\title{
Mansard Roofline Model: Reinforcing the Accuracy of the Roofs
}

DIOGO MARQUES, ALEKSANDAR ILIC, and LEONEL SOUSA, INESC-ID, Instituto Superior Técnico, Universidade de Lisboa, Portugal

Continuous enhancements and diversity in modern multi-core hardware, such as wider and deeper core pipelines and memory subsystems, bring to practice a set of hard-to-solve challenges when modeling their upper-bound capabilities and identifying the main application bottlenecks. Insightful roofline models are widely used for this purpose, but the existing approaches overly abstract the micro-architecture complexity, thus providing unrealistic performance bounds that lead to a misleading characterization of real-world ap plications. To address this problem, the Mansard Roofline Model (MaRM), proposed in this work, uncovers a minimum set of architectural features that must be considered to provide insightful, but yet accurate and realistic, modeling of performance upper bounds for modern processors. By encapsulating the retirement constraints due to the amount of retirement slots, Reorder-Buffer and Physical Register File sizes, the proposed model accurately models the capabilities of a real platform (average rRMSE of 5.4\%) and characterizes 12 application kernels from standard benchmark suites. By following a herein proposed MaRM interpretation methodology and guidelines, speed-ups of up to $5 \times$ are obtained when optimizing real-world bioinformatic application, as well as a super-linear speedup of $18.5 \times$ when parallelized.

CCS Concepts: • Computing methodologies $\rightarrow$ Modeling and simulation; $\bullet$ Computer systems organization $\rightarrow$ Architectures;

Additional Key Words and Phrases: Performance modeling, roofline modeling, application characterization

ACM Reference format:

Diogo Marques, Aleksandar Ilic, and Leonel Sousa. 2021. Mansard Roofline Model: Reinforcing the Accuracy of the Roofs. ACM Trans. Model. Perform. Eval. Comput. Syst. 6, 2, Article 7 (September 2021), 23 pages.

https://doi.org/10.1145/3475866

\section{INTRODUCTION}

To increase performance, modern multi-core Central Processing Units (CPUs) adopt deeper and wider core pipelines, with higher number of execution units [15, 33, 46]. This tendency also occurs in the memory subsystem, with the introduction of bigger caches and novel memory technologies [38-40]. Due to the ever increasing complexity that comes with the introduced enhancements, it is a challenge to uncover and exploit the maximum attainable performance of CPUs. This is imperative when pinpointing the hardware execution bottlenecks for real-world parallel

This work was supported by the FCT (Fundação para a Ciência e a Tecnologia, Portugal), ERDF (European Regional Development Fund, EU), and EuroHPC Joint Undertaking through the projects UIDB/50021/2020, LISBOA-01-0145-FEDER-031901 (PTDC/CCI-COM/31901/2017, HiPErBio), and Grants No. 956213 (SparCity) and No. SFRH/BD/136053/2018 (FCT).

Authors' address: D. Marques, A. Ilic, and L. Sousa, INESC-ID, Instituto Superior Técnico, Universidade de Lisboa, Rua Alves Redol, 9 - 1000-029 Lisboa - Portugal; emails: diogo.marques@inesc-id.pt, aleksandar.ilic@inesc-id.pt, leonel.sousa@inesc-id.pt.

This work is licensed under a Creative Commons Attribution International 4.0 License.

(c) 2021 Copyright held by the owner/author(s).

2376-3639/2021/09-ART7 \$15.00

https://doi.org/10.1145/3475866

ACM Trans. Model. Perform. Eval. Comput. Syst., Vol. 6, No. 2, Article 7. Publication date: September 2021. 
applications, which exercise compute and memory resources differently due to diverse instruction types and memory access patterns.

Recent trends to tackle these challenges rely on insightful models that abstract the complexity of the micro-architectures and applications, such as roofline approaches [9, 18, 25, 48]. The intuitiveness and usability of these models makes them powerful tools to assess the potential of computing systems, such as tensor processing units [1,22], mobile SOCs [17], sparse accelerators [36, 37], and near-memory devices [23], as well as for the characterization and optimization of applications of diverse scientific domains, e.g., physics [11, 16, 24, 43], astrophysics [7], mathematics [5, 10, 27], and chemistry [31]. Due to their insightfulness, roofline models are nowadays widely adopted even in industry profiling tools, e.g., Dynamic Random Access Memory (DRAM), Original Roofline Model (ORM) [48] in Nvidia Nsight Compute, and the Cache-Aware Roofline Model (CARM) [18] and Integrated Roofline Model (IRM) [25] in Intel Advisor.

However, the State-of-the-Art (SoA) roofline models oversimplify the back-end of the microarchitectures when modeling their performance upper bounds by focusing on a subset of functional units and by not considering components crucial to the retirement of instructions in any Out-of-Order (OoO) processor, such as the number of Retirement Slots (RS), Reorder Buffer (ROB), and Physical Register File (PRF). Instead, these models evaluate the performance upper bounds by only considering the isolated performance limits of the different hardware resources, thus giving the illusion of infinite retirement and $\mathrm{OoO}$ windows. Moreover, when concurrently executing non-memory and memory instructions, the limited capacities of ROB and PRF can constraint the number of in-flight memory requests, preventing applications from achieving maximum memory bandwidth and increasing the impact of memory latency, especially when accessing the "slower" memory levels. This effect also prevents a full overlap between memory accesses and computations, which is a main assumption of roofline models.

By not considering any of the referred bottlenecks, roofline approaches provide unrealistic model of performance upper bounds, which can lead to incorrect architecture analyses and/or misleading characterization of applications (especially the ones with a broad set of instruction types or which performance is sensitive to memory latency). This lack of accuracy affects all kinds of computing systems, from OoO CPUs to application-specific accelerator, and with the adoption and automation of traditional roofline approaches in industry frameworks for performance analysis, this issue is expected to become more relevant and severe. While some works aim at modeling a more broad set of instructions to offer roofline accuracy improvements, such as the Applicationdriven CARM proposed in Reference [30], and the Integer (INT) + Floating-Point (FP) roofline in Intel Advisor, they still inherit all previously referred roofline modeling drawbacks. To tackle these issues, the work proposed herein aims at uncovering the minimum set of micro-architectural parameters that must be considered to achieve an insightful but yet accurate roofline model. The considered parameters and methodology are general enough to be applied to any current OoO CPU, thus Mansard Roofline Model (MaRM) can be incorporated in the performance tools of any vendor of $O O O$ Central Processing Units (CPUs).

The MaRM proposed in this article models the system upper bounds from a micro-architectural point of view by considering all the instructions retired by an application, representing performance as Instructions Retired per Cycle (IPC). MaRM also takes into account the impact of the retiring limitations and constraints due to the ROB, PRF, and the number of RS, while maintaining the inherent simplicity of the roofline modeling approaches. The experimental evaluation shows that by including a more detailed modeling of the back-end of modern processors, MaRM realistic performance upper bounds allow us to correctly characterize and pinpointing the execution inefficiencies of real-world applications. Accordingly, this article includes the following set of main contributions to accurately modeling the performance of Out-of-Order $(\mathrm{OoO})$ micro-architectures: 
- a benchmarking methodology that experimentally assesses the impact of the effective ROB size under several execution scenarios, as well as the effective memory bandwidth of the system according to the ROB limitations;

- a novel roofline modeling approach, i.e., the MaRM, that provides realistic performance upper bounds of $\mathrm{OoO}$ micro-architectures by considering the constraints provoked by the number of retirement slots, Reorder Buffer (ROB) and PRF, and all the instructions retired by applications.

- validation of MaRM through the characterization of a set of applications of diverse areas, such as graph processing, machine learning and bioinformatics on a real system containing an Intel Xeon 6140 Gold.

The rest of this article is organized as follows. Section 2 motivates this work. Section 3 describes the benchmarking methods used to uncover the parameters necessary to construct the model. The MaRM model is proposed and discussed in Section 4, and Section 5 includes the experimental evaluation of MaRM. Finally, the related work and conclusions are presented Sections 6 and 7, respectively.

\section{BACKGROUND AND MOTIVATION}

To keep up with application demands, recent generations of Intel, AMD, and ARM microarchitectures tend to incorporate wider and deeper processing pipelines [33, 34, 41, 46]. For example, for the Intel Ivy Bridge to Intel Skylake-SP, the number of execution ports increased from 6 to 8. Together with double the vector instruction width (from 256-bit to 512-bit), these enhancements led to improved performance [2]. Other components, such as the ROB and the PRF, also had their sizes increased, allowing for higher $\mathrm{OoO}$ and retirement windows. Following this trend, the next Intel server architecture, the Intel IceLake-SP, provides 10 execution ports, wider retirement window, and extended ROB, PRF, and caches [33].

When modeling the micro-architecture performance, roofline models usually focus on the FP SIMD instructions that achieve maximum computational performance and memory bandwidth, e.g., FP AVX512 in Intel Skylake-SP, while abstracting the remaining functional units contained in the core back-end. As such, these models relate the system performance upper bounds with the Arithmetic Intensity (AI), i.e., the number of flops performed over the amount of bytes transferred, by assuming that memory transfers and computations are executed concurrently, due to the $\mathrm{OoO}$ engine of multi-core CPUs. While the computational units are evaluated equally in all roofline approaches, these models take a different perspective of the memory hierarchy. While CARM [18] and IRM [25] encapsulate the maximum sustainable bandwidths between the core and each memory level "y" ( $y \in\{L 1, L 2, \ldots, L L C, D R A M\})$, ORM and hORM $[9,48]$ consider the maximum bandwidth between two consecutive memory levels.

While the simplicity of current roofline models results in powerful tools to easily obtain insights regarding the execution bottlenecks of applications, it can become a drawback when evaluating micro-architecture performance and characterizing real-world workloads. By only focusing on specific instruction types instead of all the instructions retired by an application, traditional roofline approaches are unable to accurately model the performance of real-world applications that exercise diverse units of the micro-architectures during execution.

Furthermore, roofline models do not consider the number of RS in the micro-architecture, implying an infinite retirement window. By considering the isolated performance of the different execution units, these models very likely overestimate the performance upper bounds of a system. The performance upper bounds of recent Intel micro-architectures according to roofline approaches are presented in Figure 1(a). As can be observed, roofline models predict an IPC always superior to the number of RS. In fact, when maximizing the utilization of the memory ports, i.e., 2 loads and 1 


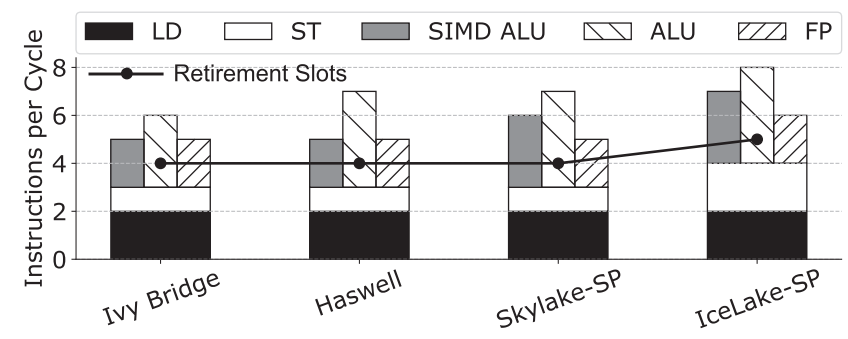

(a) Retirement slots.

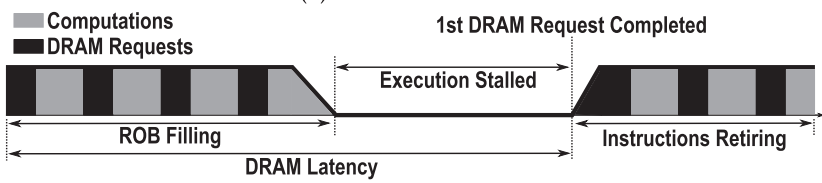

(b) Reorder buffer.

Fig. 1. Effect of the Retirement Slots and Reorder Buffer in performance upper bounds.

store per cycle, this effect occurs always independently of type of computations performed. This indicates that although memory transfers and computations can be executed simultaneously, the performance is limited by the retirement window.

Another limitation of roofline models is related to the effective size of the ROB. Figure 1(b) shows that when accessing a high latency memory level (in this example DRAM) its latency far exceeds the time that takes to fill the $\mathrm{ROB}$, stalling the processor until the memory request is satisfied. When interleaving memory requests and computations, this prevents those instructions to be retired concurrently, and application execution becomes highly dependent on the latency. Furthermore, since the effective bandwidth and latency depend on the number of in-flight memory requests, when interleaving memory accesses and computations the effective size of the ROB also limits the micro-architecture from achieving the maximum sustainable bandwidth of the DRAM. The load and store buffers can also play an important role when limiting the bandwidth of the system, especially when there are dependencies between memory instructions. However, when evaluating system upper bounds their impact is minimal, since memory accesses are typically independent of each other.

\section{MICRO-ARCHITECTURE BENCHMARKING}

To evaluate the effects of the discussed retiring issues in the roofline models, two benchmarks typically used to validate the SoA roofline models are executed ${ }^{1}$ in a system containing the Intel Xeon Gold 6140 processor presented in Figure 2. The structure of the benchmark for L1 cache validation with Advanced Vector Extension (AVX) loads and AVX 32-bit INT Additions (ADD) computations is presented in Figure 3(a). This benchmark operates over an array that only fits in the tested memory level (in this case, L1 cache) and interleaves memory accesses and computations. The amount of memory instructions interleaved with the computations is defined by the ridge point, i.e., the machine balance, which corresponds to the peak performance of the computations over the maximum sustainable bandwidth of the memory level. Since the peak performance of AVX INT 32-bit ADD is 993.6 GINTOP/s and the maximum sustainable bandwidth of L1 cache for AVX instructions is $2,773 \mathrm{GiB} / \mathrm{s}$ (see Figure 2), the benchmark should approximately have 1.5

\footnotetext{
${ }^{1}$ The experimental results presented in this work correspond to the median of 1,024 executions and are performed under the setup and by using the performance counters presented in Figure 2.
} 


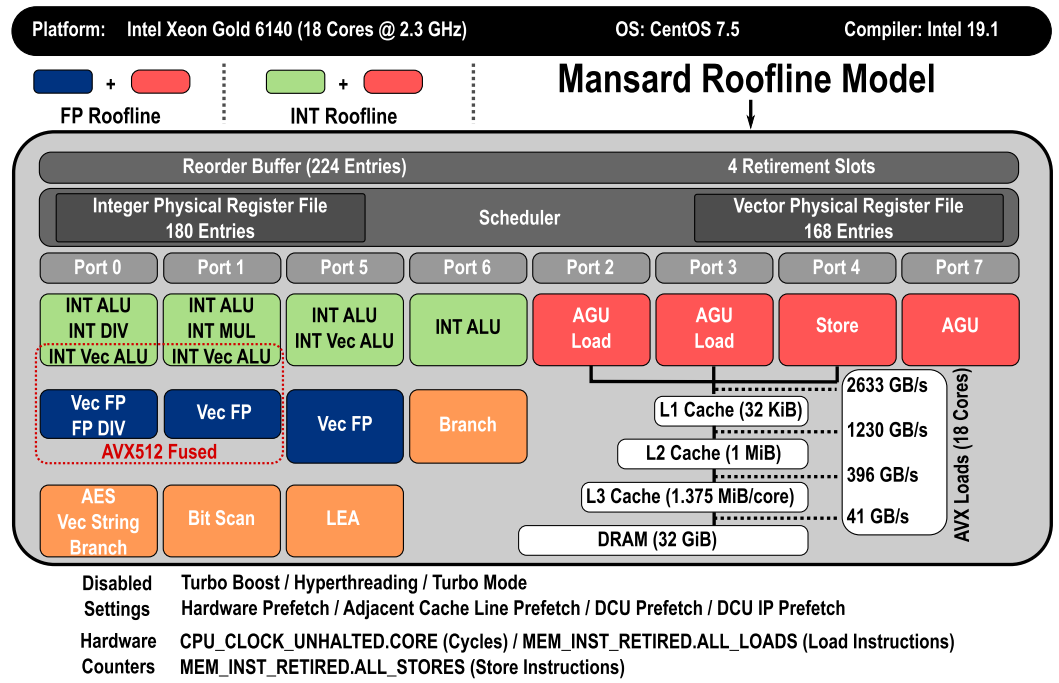

Fig. 2. Backend of the system used in this work.

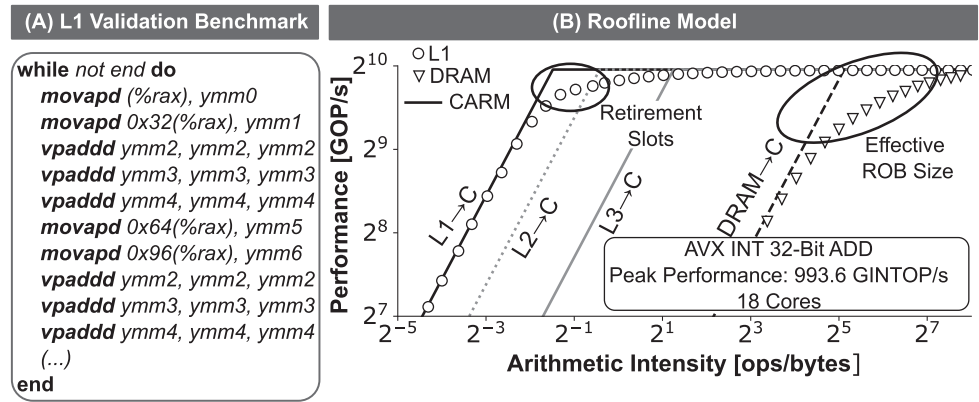

Fig. 3. Impact of retiring issues in roofline models.

compute instructions per memory access. The same methodology is used for Dynamic Random Access Memory (DRAM) by using an array bigger than L3 cache and by interleaving memory and compute instructions according to the machine balance of DRAM.

The validation results for the SoA roofline models are presented in Figure 3(b). As it can be observed, around the ridge points of L1 cache and DRAM, it is not possible to achieve the maximum performance predicted by the roofline models. For L1 cache, the roofline models consider that both memory instructions and computations can retire at its maximum throughput, i.e., IPC = 2 for loads and Instructions Retired per Cycle (IPC) = 3 for INT AVX computations, predicting a maximum IPC of 5 for this mix of instructions. However, Skylake-SP only has four retirement slots, which limits the maximum dispatch rate of the micro-architecture. In the case of performing 2 loads and 1 store per cycle, this difference would be more evident. Regarding DRAM, the issue is even more severe. The inaccuracy provided by the roofline models arises from limited ROB size, which in this case restricts the number of in-flight requests and increases the impact of the DRAM latency. For the Skylake-SP and AVX loads, the maximum sustainable bandwidth of DRAM is $41 \mathrm{~GB} / \mathrm{s}$, thus when interleaving memory requests and AVX INT 32-bit ADD instructions, the ROB contains approximately 97 compute instructions per memory request. Since the ROB of SkylakeSP has 224 entries, only 3 memory requests can be performed simultaneously $(224 /(97+1))$, which 


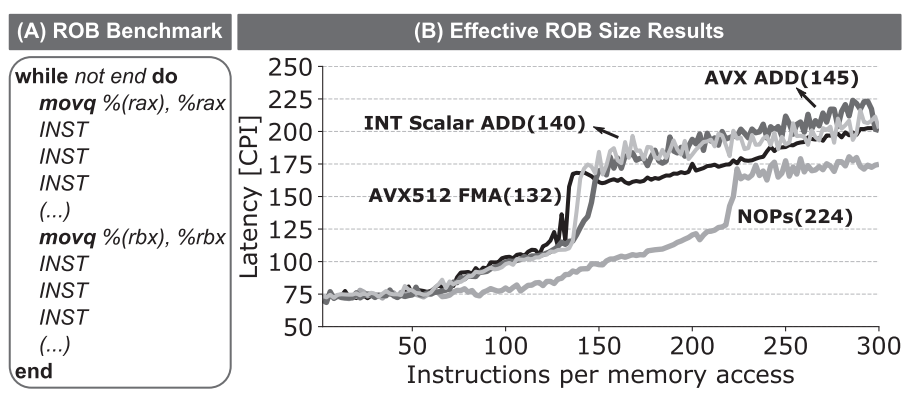

Fig. 4. Effective ROB size for different instructions.

is not enough to fully hide DRAM latency. From this analysis, it is possible to conclude that SoA roofline approaches can provide inaccurate characterization and guidelines, by giving the illusion that applications can achieve the maximum performance predicted by the models, when in fact it is impossible to reach those regions.

To achieve an accurate roofline model, the MaRM must include instruction retirement limits of multi-core CPUs. Although real-world applications contain different instruction types, which enable them to make full usage of the ROB capacity, applications completely dominated by a single instruction type, e.g., vector instructions, will be limited by the PRF capacity. Hence, while the number of retirement slots can be easily obtained from data-sheets, assessing the effective size of the $\mathrm{ROB}\left(R O B_{\text {eff }}\right)$ is only possible through micro-benchmarks that stress the PRF under different execution scenarios. Moreover, since the $R O B_{e f f}$ constraints the number of in-flight memory requests $\left(I F_{M}\right)$, when memory and compute instructions are interleaved, it influences the effective memory bandwidth of the system. Thus, it is also necessary to experimentally assess the bandwidth variation with the $I F_{M}$. While in this work the benchmarking methodology focuses on in-flight memory requests and PRF, it is flexible enough to evaluate the impact of other components and bottlenecks, such as the load and store queues, non-temporal stores and other cache-bypassing operations.

\subsection{Uncovering the Effective Reorder-Buffer Size}

To expose the impact of PRF capacity in the $R O B_{\text {eff }}$, a set of micro-benchmarks were developed, adopting the methodology proposed in Reference [49]. As depicted in Figure 4(a), these tests consist of two independent pointer chases separated by a certain amount of instructions (INST). These instructions change accordingly to the hardware component to test. For example, No-Operation (NOP) instructions are used to experimentally assess the capacity of the ROB, while vector Floating-Point (FP) instructions allow to determine the impact of the vector FP PRF in the ROB. When the two memory instructions fit in the ROB, their execution is performed concurrently, thus the latency is approximately half of the latency of a single pointer-chasing. Once the amount of instructions per memory access exceeds the effective ROB size, the instruction movq $\%(r b x), \% r b x$ will not fit in the ROB, provoking a significant increase in the average memory latency, since the memory accesses are no longer being executed simultaneously. This allows to identify the effective size of the ROB under different execution scenarios. To avoid the access to different cache levels, the pointer chasing arrays are initialized with addresses separated by $64 \mathrm{~B}$ (cache line size). For the tests that use the PRF, all the possible assembly registers are used to avoid dependencies, and to maximize the utilization of the PRF.

The experimental evaluations of the effective ROB size for NOP , 512-bit-AVX (AVX512) Fused Multiply-Add (FMA) instructions, AVX ADD instructions and scalar integer additions (INT ADD) are presented in Figure 4(b). Since NOP do not use any register during execution, the memory 


(A) Bandwidth Test
while no end do
MEM
MEM
MEM
MEM
$(\ldots)$
NOP
NOP
NOP
(...)
end
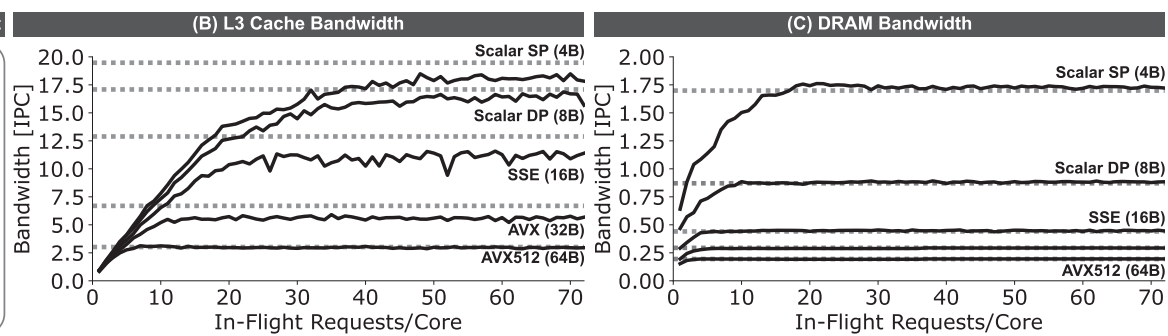

Fig. 5. Bandwidth variation with the amount of concurrent requests for $\mathrm{L} 3$ and DRAM and 2LD/ST ratio.

latency drastically increases approximately at the size of the ROB, i.e., 224 entries. For AVX512 FMA instructions, the memory latency increases when there are 132 instructions per memory access, i.e., around $78.6 \%$ of the vector PRF capacity (168 entries), while for Advanced Vector Extension (AVX) ADD instructions, the effective ROB size is close to 145 entries. This non-ideal behavior indicates the existence of additional bottlenecks in different components of the core pipeline. The results also show that the effective $\mathrm{ROB}$ size depends on the instruction used when accessing a register file, as it is the case of the AVX512 FMA and AVX ADD instructions. Similar scenario occurs for the INT ADD test, with an effective ROB size around 140 entries.

\subsection{Relating Bandwidth and In-flight Memory Requests}

To relate the effective micro-architecture bandwidth and the number of in-flight memory requests, the developed benchmarking exploits the organization of instructions in the ROB, by managing the number of memory requests that are simultaneously in this component. As it can be observed in Figure 5(a), the test consists of two main blocks. The first block is composed only of memory instructions, which correspond to the number of in-flight requests and perform sequential accesses over an array that fits only in the tested memory level. The second block only contains NOP, to avoid constraints in the ROB through exhaustive utilization of other components of the core pipeline (e.g., PRF). The sum of memory and NOP instructions is always equal to the ROB size, to guarantee the control over the amount of memory instructions contained in the ROB. This methodology can be applied to diverse ratios of loads (LD) and stores (ST) (LD, 2LD/ST, LD/ST, and ST) and data sizes (64B, 32B, etc.).

The results obtained for L3 cache and DRAM, considering 2LD/ST ratio, and different data sizes are presented in Figure 5. All the curves in this figure have similar characteristics. For small amount of in-flight memory requests, the bandwidth increases in a non-linear fashion. Once the number of concurrent accesses is high enough to fully hide the memory latency, the bandwidth converges to the maximum sustainable bandwidth, corresponding to the dashed horizontal lines. For example, for the DRAM test with Scalar Double-Precision (DP) instructions, the effective bandwidth for 1 in-flight group of $2 \mathrm{LD}+1 \mathrm{ST}$ per core is around 0.5 , while the maximum sustainable bandwidth is attained around 10 in-flight groups of $2 \mathrm{LD}+1 \mathrm{ST}$ per core, converging to an IPC around 0.9. In the scenario, SoA roofline models would overestimate DRAM bandwidth approximately by $2 \times$. It is also possible to verify that the bandwidth and the number of in-flight requests vary with the data size. For example, for L3 test the AVX512 instructions attain the maximum sustainable bandwidth of 2.95 around 7 groups of concurrent 2LD + 1ST requests, while for Scalar SP instruction it attains an IPC of 18.85 only when 48 groups of 2LD + 1ST are simultaneously in the ROB.

The relation between the effective memory bandwidth and the number of in-flight memory requests can also be derived from the bandwidth-latency curves [19], by applying the Little's Law [28] to each of its points. In the related SoA, this curve is usually obtained by executing one 


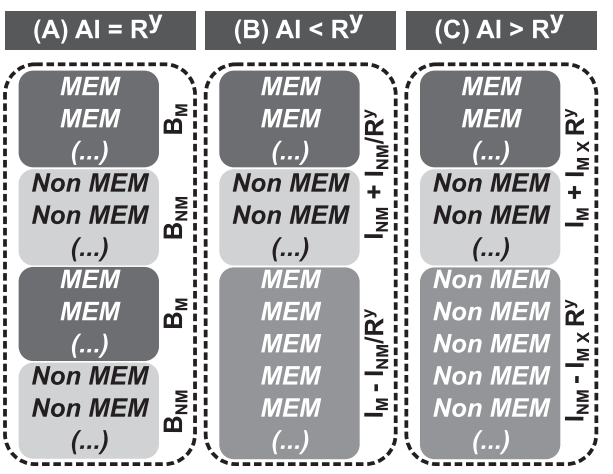

Fig. 6. Execution scenarios for different application types.

pointer-chasing benchmark in one thread, while the remaining $\mathrm{N}-1$ threads perform sequential accesses to the memory. To simulate latency variations, a delay is injected between the execution of the two experiments. By measuring the latency of the pointer chasing and the attained bandwidth of the N-1 threads, it is possible to relate both parameters. However, with this methodology, the curve is constructed for the bandwidth of $\mathrm{N}-1$ threads instead of the sustainable bandwidth of the entire system. Moreover, this method only provides results when all threads need to access the memory level exactly through the same hardware components. Hence, this approach is not adequate to evaluate the L3 cache, since each thread accesses this memory through its own slice, and the latency thread is not affected by the $\mathrm{N}-1$ bandwidth threads.

\section{MANSARD ROOFLINE MODEL}

To address the main drawbacks of the SoA roofline models, the MaRM considers all the instructions retired by an application, representing performance as IPC. Since MaRM uses the instruction domain instead of the operations domain, its AI differs from standard roofline models. In MaRM, the $\mathrm{AI}$ is defined as the number of non-memory instructions $\left(I_{N M}\right)$ over the number of memory instructions $\left(I_{M}\right)$. Thus, the ridge point of memory level "y" $\left(R^{y}\right)$, i.e., the point where memory transfers and computations are completely overlapped in time, is represented as the performance of the non-memory instructions $\left(I P C_{N M}\right)$ over the maximum sustainable bandwidth of memory level "y" $\left(I P C_{M, \text { Max }}^{y}\right)$.

For representing performance as IPC, it is indispensable to incorporate the retirement limits of OoO CPUs described previously. Due to the limited number of retirement slots, micro-architectures can only retire a limited number of instructions per cycle. Hence, the maximum attainable performance $\left(P_{a}^{y}(A I)\right)$ is limited by the maximum dispatch rate $\left(D_{M}\right)$ of the micro-architecture such that

$$
P_{a}^{y}(A I) \leq D_{M},
$$

where $D_{M}$ is usually 4 instructions per cycle in a core with current Intel micro-architectures.

To include the impact of the ROB in MaRM, the three execution scenarios in Figure 6 are considered. These execution scenarios are representative of applications or application kernels with different AIs (expressed in $\frac{I_{N M}}{I_{M}}$ ), which according to roofline approaches are able to attain the maximum performance in different regions of the models. Scenario A fully overlaps memory and non-memory instructions, i.e., ArithmeticIntensity(AI) $=R^{y}$; in Scenario B, applications are memory bound $\left(\mathrm{AI}<R^{y}\right)$; and Scenario $\mathrm{C}$ portrays workloads in the compute bound region $\left(\mathrm{AI}>R^{y}\right)$. 
At the ridge point (scenario $\mathrm{A}$ ), the ROB has several blocks containing $B_{M}$ memory instructions and $B_{N M}=B_{M} \times R^{y}$ non-memory instructions. For example, an application with an average of 2 loads per store has $B_{M}=3$ and $B_{N M}=3 R^{y}$. Since the amount of in-flight memory requests $\left(I F_{M}\right)$ corresponds to the total number of memory instructions in the ROB, $I F_{M}$ can be calculated as

$$
I F_{M}=B_{M} \times\left\lceil\frac{R O B_{e f f}}{B_{M}+B_{N M}}\right\rceil=B_{M} \times\left\lceil\frac{R O B_{e f f}}{B_{M} \times\left(R^{y}+1\right)}\right\rceil,
$$

where $R O B_{\text {eff }}$ is the effective $\mathrm{ROB}$ size, which can be obtained with the methodology proposed in Section 3.

When accessing high latency memory levels, the blocks $B_{M}+B_{N M}$ that fit in the ROB can only start retiring after the first memory request is completed. Thus, the execution time of the blocks $B_{M}+B_{N M}$ contained in the ROB is approximately the latency of the corresponding memory level "y" $\left(\operatorname{Lat}^{y}\left(I F_{M}\right)\right)$, thus the performance of the micro-architecture at the ridge point $\left(\operatorname{IPC}_{R}^{y}\left(I F_{M}\right)\right)$ is given by

$$
\begin{aligned}
\operatorname{IPC}_{R}^{y}\left(I F_{M}\right) & \approx \frac{I F_{M} \times\left(R^{y}+1\right)}{\operatorname{Lat}^{y}\left(I F_{M}\right)} \\
& =\left(R^{y}+1\right) \times I P C_{M}^{y}\left(I F_{M}\right),
\end{aligned}
$$

where $I P C_{M}^{y}\left(I F_{M}\right)$ is the effective memory bandwidth of the level "y" restricted by the number of in-flight requests. This parameter is related to $\operatorname{Lat}^{y}\left(I F_{M}\right)$ through Little's Law $\left(\operatorname{IPC}_{M}^{y}\left(I F_{M}\right) \times\right.$ $\left.L a t^{y}\left(I F_{M}\right)=I F_{M}\right)$ [28]. The effect modeled by Equation (3) corresponds to the illustration presented in Figure 1(b).

Memory bound (scenario B) and compute-bound (scenario C) applications also aim at overlapping non-memory and memory instructions, but since their $\mathrm{AI}$ is different from $R^{y}$ they are made up of two components each. The first one overlaps memory and non-memory instructions in the same fashion as scenario A. The second component only contains instructions of a single type. Since in scenario B the $\mathrm{AI}$ is lower than $R^{y}$, there are more memory instructions than non-memory instructions. Thus, the first component contains all non-memory instructions overlapped with $\frac{I_{N M}}{R^{y}}$ memory transfers, corresponding to a total of $I_{N M}+\frac{I_{N M}}{R^{y}}$ instructions, while the second component contains the remaining memory instructions, i.e., $I_{M}-\frac{I_{N M}}{R^{y}}$. Since the overlapping component is organized as in scenario $\mathrm{A}$, it is executed at the rate of $\operatorname{IPC}_{R}^{y}\left(I F_{M}\right)$. In the second component, instructions are retired at the maximum sustainable memory bandwidth $\left(I P C_{M, M a x}^{y}\right)$, since there are enough requests in the $\mathrm{ROB}$ to attain the maximum bandwidth. Hence, the number of cycles necessary to execute a memory-bound application $\left(C y c_{B}\right)$ is given by

$$
\begin{aligned}
C y c_{B}^{y}\left(A I, I F_{M}\right)= & \frac{I_{N M}+\frac{I_{N M}}{R^{y}}}{I P C_{R}^{y}\left(I F_{M}\right)}+\frac{I_{M}-\frac{I_{N M}}{R^{y}}}{I P C_{M, \text { Max }}^{y}} \\
& =\frac{A I \times I_{M}\left(1+\frac{1}{R^{y}}\right)}{I P C_{R}^{y}\left(I F_{M}\right)}+\frac{I_{M} \times\left(1-\frac{A I}{R^{y}}\right)}{I P C_{M, \text { Max }}^{y}} .
\end{aligned}
$$

However, the first code portion of compute-bound applications (scenario C) contains all memory instructions overlapped with $I_{M} \times R^{y}$ non-memory instructions, i.e., a total of $I_{M}+I_{M} \times R^{y}$ instructions, retired at the speed of $I P C_{R}^{y}\left(I F_{M}\right)$. The second component contains $I_{N M}-I_{M} \times R^{y}$ nonmemory instructions retiring at the performance of the non-memory instructions $\left(I P C_{N M}\right)$. Thus, the number of cycles necessary to execute the application $\mathrm{C}(\mathrm{Cyc} C)$ can be calculated as follows: 


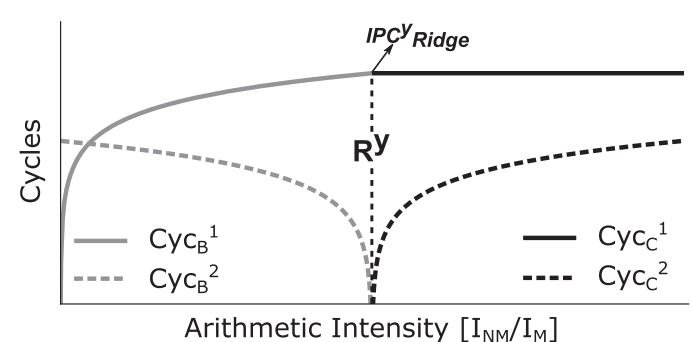

Fig. 7. Illustration of cycles of the proposed MaRM.

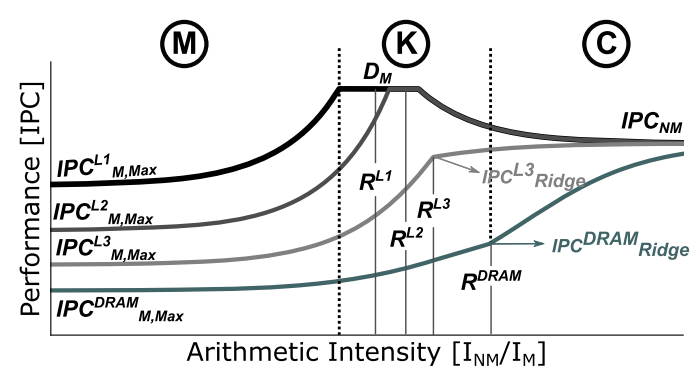

Fig. 8. Illustration of MaRM.

$$
\begin{aligned}
\operatorname{Cyc}_{C}^{y}\left(A I, I F_{M}\right) & =\frac{I_{M}+I_{M} \times R^{y}}{I P C_{R}^{y}\left(I F_{M}\right)}+\frac{I_{N M}-I_{M} \times R^{y}}{I P C_{N M}} \\
& =\frac{R^{y} \times I_{M}\left(1+\frac{1}{R^{y}}\right)}{I P C_{R}^{y}\left(I F_{M}\right)}+\frac{I_{M} \times\left(\frac{A I}{R^{y}}-1\right)}{I P C_{M, \text { Max }}^{y}} .
\end{aligned}
$$

Equations (4) and (5) are illustrated in Figure 7, each represented by their respective components, i.e., $C y c_{B}^{1}$ and $C y c_{B}^{2}$ from $C y c_{B}^{y}\left(A I, I F_{M}\right)$, and $C y c_{C}^{1}$ and $C y c_{C}^{2}$ from $C y c_{C}^{y}\left(A I, I F_{M}\right)$. There is a relation between the components of both equations. Regarding the first components, $C y c_{C}^{1}$ is always constant and equal to $C y c_{B}^{1}$ at the ridge point. Thus, from the analysis of Figure 7, Equations (4) and (5) can be unified in a single equation such that the application execution time $\left(C y c_{A p p}\right)$ is given by

$$
C y c_{A p p}=I_{M} \times\left(\frac{\min \left(A I, R^{y}\right)\left(1+\frac{1}{R^{y}}\right)}{I P C_{R}^{y}\left(I F_{M}\right)}+\frac{\left|\frac{A I}{R^{y}}-1\right|}{I P C_{M, M a x}^{y}}\right) .
$$

Finally, from Equations (1) and (6), it is possible to derive the MaRM performance $\left(\operatorname{IPC}_{a}^{y}\left(A I, I F_{M}\right)\right)$ as follows:

$$
I P C_{a}^{y}\left(A I, I F_{M}\right)=\min \left(\frac{I_{M}+I_{N M}}{C y c_{A p p}}, D_{M}\right) .
$$

Figure 8 illustrates the proposed MaRM. As it can be observed, MaRM includes in the memorybound region the entire memory hierarchy, with each level represented by their maximum sustainable bandwidth. The compute-bound region of the model is limited by the performance of the non-memory instructions. In contrast to State-of-the-Art (SoA) roofline models, the roofs in MaRM have a format similar to a "hill," and the memory roofs are no longer diagonal lines. Since MaRM performance cannot surpass the maximum retirement rate of the micro-architecture, flat 
regions may occur in the model, indicating areas where the application is limited by the number of retirement slots. This is observed for the L1 roof in Figure 8. While in CARM the ridge point corresponds to the minimum $\mathrm{AI}$ that allows attaining maximum performance for any memory levels, in MaRM this does not occur for L3 and DRAM. Due to the high latency of these memory levels, their effective bandwidth depends on the amount of concurrent memory requests. Thus, the ridge points of the L3 cache and DRAM do not correspond to the point where maximum performance is achieved when accessing those memory levels. In fact, the performance continues to increase beyond the ridge point due to the growing contribution of the compute instructions, asymptotically approaching to the maximum performance of the compute units. However, for L1 and L2 caches, the micro-architecture is able to attain the maximum sustainable bandwidth for the entire range of AI, until reaching the ridge point. Hence, the ridge point in MaRM inherits the properties of Cache-Aware Roofline Model (CARM) for L1 and L2 caches.

\subsection{Discussion}

Equation (7), which analytically defined the MaRM, considers all the instructions retired by an application and encapsulates the performance bottlenecks that arise from the retirement limits of the OoO micro-architecture. In fact, by analyzing MaRM equations, the proposed instructionbased model can be regarded as a generalization of the CARM. By assuming an infinite ROB, this component does not limit the amount of in-flight memory requests neither it stalls the processor. In this scenario, $I P C_{\text {Ridge }}^{y}\left(I F_{M}\right)$ is no longer dependent on $I F_{M}$ and $I P C_{M}^{y}\left(I F_{M}\right)=I P C_{M, \text { Max }}^{y}=I P C_{M}^{y}$. In this scenario, $C y c_{A p p}$ is given by

$$
C y c_{A p p}=\frac{I_{M} \times\left|A I-R^{y}\right|}{I P C_{N M}}+\frac{\min \left(A I, R^{y}\right) \times I_{M}}{R^{y} \times I P C_{M}^{y}} .
$$

By considering the intervals $A I \leq R^{y}$ and $A I>R^{y}$ and that $I P C_{N M} / I P C_{M}^{y}=R^{y}$, the execution time can be described as

$$
C y c_{A p p}= \begin{cases}\frac{I_{M}}{I P C_{M}^{y}}, & A I \leq R^{y} \\ \frac{I_{N M}}{I P C_{N M}}, & A I>R^{y}\end{cases}
$$

which corresponds to CARM execution time [18]. From Equation (9), it is possible to conclude that CARM is a specific case of MaRM, when it is assumed maximum sustainable bandwidth for the entire range of AI and for all levels of the memory hierarchy. This analysis also provides an analytical proof that CARM overestimates the performance upper bounds.

To tradeoff modeling accuracy and simplicity, some assumptions are considered when constructing MaRM. In particular, for the calculation of the $I F_{M}$ parameter, it is assumed that a single ROB entry is requested per instruction. For the Intel case, while there are instructions that use more than a single entry, the macro-fusion and micro-fusion performed during the decoding of the microoperations prevent this effect from occurring frequently for the instructions that belong to the most recent instruction set architecture extensions. Moreover, the instructions that match the performance upper bounds of modern Intel systems do only occupy a single ROB entry, hence this is an acceptable assumption for deriving MaRM.

In the micro-architecture evaluated in this work, the latencies of L1 and L2 caches are small compared to the ones of L3 cache and DRAM. Thus, when considering independent memory accesses, which are used to evaluate the bandwidth upper bounds, L1 and L2 caches are unlikely to stall the processor and the effective size of the ROB does not prevent the micro-architecture from achieving the maximum sustainable bandwidth. For this reason, in the proposed model, the maximum sustainable bandwidth is adopted for L1 and L2 caches. However, this assumption depends 
on the tested micro-architecture, and in the case that one of these memory levels causes the ROB issues considered in MaRM, the methodology proposed in Section 3 can be applied.

Since MaRM takes into account all instructions supported by a given CPU, it is expected to correctly classify the bottlenecks of the applications containing varied instruction types, from FP and INT to logical and special instructions (e.g., shifts, converts). In this scenario, it is not possible to define computational roofs for specific types of instructions. Instead, MaRM computational roofs correspond to the main retirement rates of the micro-architecture according to the number of Retirement Slots (RS), i.e., in the range of one retirement slot (1RS) to four retirement slots (4RS) per core in current Intel CPUs.

Finally, the MaRM AI does not inherit the properties and limitations of the CARM counterpart. In CARM, AI is considered a property of the algorithm and it is expected to remain constant throughout the application optimization process (unless the algorithm structure is modified). However, when referring to instructions, depending on the optimization techniques used, the number of non-memory and memory instructions may scale differently. For example, an optimization that vectorizes all the non-memory instructions but barely affects the memory accesses will decrease the relative number of non-memory instructions (overall, the number of computations and bytes is not affected). Thus, in MaRM it is possible to move AI of applications, from the compute region to the memory region and vice versa, along the optimization process.

\subsection{Model Interpretation}

As it can be observed in Figure 8, MaRM contains three main regions: memory region, where application performance is limited by the memory bandwidth of each memory level; compute region, delimited by the maximum retirement rate of the system; and mixed region (K), where the bottlenecks can be either related to the memory accesses or the maximum achievable performance. The bottleneck identification is performed by plotting a vertical line at the application AI. The intersections right above and below the application dot correspond to the main sources of inefficiencies. Depending on the region where the application is located, a set of optimizations can be derived to improve the execution time. In the memory region, the optimization should focus on improving the memory accesses, while in the compute region vectorization methods can be employed to improve application execution. In the mixed region, techniques from both memory and compute regions might be used according to the bottlenecks identified at each optimization phase.

In the mixed region of the roofs correspondent to the "slower" memory levels, applications are expected to be limited by both memory bandwidth and latency. Compared to the SoA roofline models, this property is exclusive to MaRM, and arises from the ROB impact to the bandwidth of the memory subsystem and retirement of instructions. This effect becomes more relevant as the AI approaches the ridge point. Moreover, since MaRM represents performance as IPC, the model is oblivious to the vector width. For this reason, certain optimizations lead to lower execution time with reduced IPC. For example, in current Intel micro-architectures, an application heavily dominated by scalar INT instructions may be closer to the retirement roof of 4RS. However, by vectorizing the INT instructions, the IPC of the application will tend to the roofs correspondent to the retirement rate of SIMD ALU (3RS in most recent Intel CPUs).

Another scenario is the optimization of a compute-bound application dominated by Scalar instructions. This workload will be limited by the horizontal roof corresponding to the performance of these instructions (for example, 2RS for FP scalar instructions), indicating that there is no room for optimization, although the application can be easily sped-up by using vector instructions. Thus, when a scalar application is already on top of the computational roof that resembles the maximum throughput that its instruction mix can achieve, the recommendation is to attempt the code vectorization. 

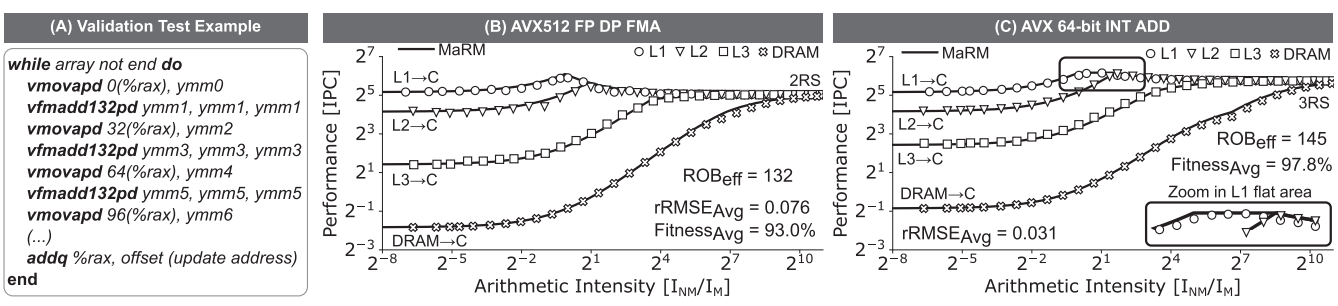

Fig. 9. MaRM validation with loads for an Intel Xeon Gold 6140 (18 Cores).

Table 1. List of Application Kernels Characterized in SoA CARM and MaRM

\begin{tabular}{|c|c|c|c|c|c|c|c|}
\hline Application & Benchmark Suite & Input & (\#Kernel) Name & Application & Benchmark Suite & Input & (\#Kernel) Name \\
\hline PR & & & (1) Function at pr.cc: 40 & SSSP & GAP & Twitter & (1) Loop at sssp.cc:106 \\
\hline \multirow[t]{2}{*}{$\mathrm{BC}$} & GAP & Twitter & (1) Loop at bc.cc: 122 & $\begin{array}{l}\text { Sparse Dense } \\
\text { Matrix Mult. } \\
\text { (SpDMM) }\end{array}$ & MKL & $\begin{array}{l}\text { BSR, } \\
N=8192, \\
\text { Spar- } \\
\text { sity }=25 \%\end{array}$ & (1) Function mkl_sparse_d_mm \\
\hline & & & (2) Loop at bc.cc: 67 & $\begin{array}{l}2 \mathrm{D} \\
\text { Convolution } \\
\text { (2D Conv.) }\end{array}$ & $\begin{array}{l}\text { Intel } \\
\text { Caffe/MKL } \\
\text { DNN }\end{array}$ & $\begin{array}{l}\text { ResNet50 } \\
\text { Inference }\end{array}$ & (1) Function convolution_forward \\
\hline BFS & & & (1) Function at bfs.cc:70 & & & & (1) Loop at HJM_SimPath_Forward_Blocking.cpp:65 \\
\hline $\mathrm{CC}$ & & & $\begin{array}{l}\text { (1) Loop at cc.cc: } 105 \\
\text { (2) Loop at cc.cc: } 133\end{array}$ & Swaptions & Parsec 3.0 & Native & $\begin{array}{l}\text { (2) Loop at HJM_SimPath_Forward_Blocking.cpp: } 116 \\
\text { (3) Loop at HJM_SimPath_Forward_Blocking.cpp: } 144\end{array}$ \\
\hline
\end{tabular}

While the drop in IPC might look counter intuitive, it increases MaRM intuition regarding the ability to evaluate the vectorization efficiency. For example, when the throughput of scalar ad vector instructions is equal (e.g., FP AVX512), three scenarios can occur after vectorization: (1) IPC remains constant, indicating that the vectorization attained maximum efficiency and the execution time reduced proportionally to the vector size; (2) the IPC reduced, hinting that the contribution of memory accesses and/or vectorization overheads increased and the vectorization did not attain $100 \%$ efficiency; and (3) the IPC is equal to 1 /vector_width, corresponding to the worst case scenario where the vectorization did not resulted in any benefit and the execution time remains almost the same.

\section{EXPERIMENTAL RESULTS}

To verify the correctness and the accuracy of the proposed MaRM, a validation of the model is performed for the processor in Figure 2 (Section 3). Furthermore, a set of applications from diverse research areas, such as graph processing and machine learning, are characterized in MaRM and compared with the characterization provided by the SoA CARM, and by the Top-Down method [50]. Finally, a guided optimization example is also provided, to demonstrate the capabilities of MaRM over SoA roofline models when optimizing applications. The experimental evaluation is performed under the same setup used for the micro-architecture benchmarking.

Besides the set of performance counters used in the micro-architecture benchmarking, it is also necessary to rely on the INST_RETIRED_ANY counter to get the amount of retired instructions. To estimate the amount of non-memory instructions, the amount of loads and stores is subtracted from the total amount of retired instructions. Moreover, Intel Software Development Emulator (SDE) v.8.50 [42] is used to identify the amount of mixed instructions (non-memory instructions with at least one memory operand) and correct the number of non-memory and retired instructions. This correction is necessary as the INST_RETIRED_ANY only counts the last micro-operation retired by an instruction. Hence, non-memory instructions with at least one memory operand (mixed instructions), will only count as one instruction, despite the fact that they should be counted as one non-memory instruction and one memory instruction (due to the memory operand). Intel SDE is also used to obtain a breakdown of the data size of the memory transfers to scale the 


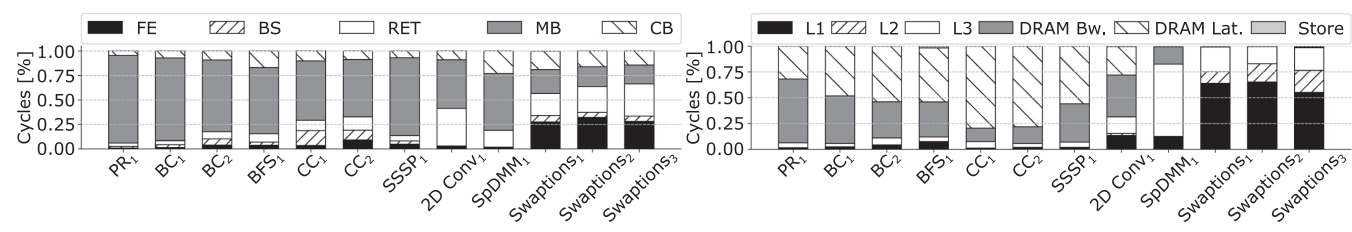

(a) 1st level breakdown.

(b) Memory bound (MB) breakdown.

Fig. 10. Top-Down method.

bandwidth roofs according to application specifics, by following the methodology presented in Reference [30]. This methodology provides bandwidth upper bounds that resemble application characteristics, aiming at providing a more accurate characterization of workloads.

\subsection{Model Validation}

The micro-benchmarking structure presented in Figure 9(a) was used to validate the MaRM. The micro-benchmark interleaves independent non-memory instructions and memory instructions to avoid dependencies, and execute them as concurrently as possible. Due to the highly flexible structure of the validation benchmarks, they can be used to validate any memory level, by changing the size of the accessed array and by interleaving memory and non-memory instructions according to the ratio of their performances. The AI is controlled by varying the amount of non-memory instructions. These tests are also the only benchmarks that allow attaining maximum system performance across all the range of AI and they are also used to validate current roofline models.

The validation results for 18 cores, and interleaving AVX512 loads with AVX512 FP DP FMA, and AVX loads AVX 64-bit INT ADD instructions are presented in Figure 9(b) and Figure 9(c), respectively. As it can be observed, MaRM is successfully validated for different execution scenarios, attaining an average $\mathrm{rRMSE}^{2}$ of 0.0535 across all the tests. In particular, an average rRMSE of 0.076 is obtained for the AVX512 FP DP FMA test, and 0.031 for the AVX 64-bit INT ADD test. The validation results confirm that the MaRM accurately considers the retirement limits of the current $\mathrm{OoO}$ micro-architectures, in particular the L3 and DRAM roofs limitations due to the effective size of the $\mathrm{ROB}$ and the maximum retirement rate, which can be observed in the zoom of the flat region of the L1 roof in Figure 9(c).

\subsection{Application Characterization}

To verify the usability of MaRM, a set of applications from diverse areas are characterized in the MaRM and in the SoA CARM. The applications in Table 1 belong to four different benchmark suite/libraries, namely GAP Benchmark Suite [4], Intel MKL [47], Intel Caffe/MKL DNN [20] and Parsec 3.0 [3]. The applications were all compiled with Intel Compiler v19.1, with the flag -xCOMMON-AVX512, to use the AVX512 vector instructions, and executed with 18 threads, each bound to a single core. The analysis is focused on the kernels that consume most of the execution time, identified with the Intel Advisor analysis. Since INT + FP Advisor CARM considers both INT and FP operations, it is the closest CARM modeling approach to MaRM. For this reason, this model is selected for comparison against MaRM, with the memory and FP roofs scaled accordingly to Reference [30]. Most of the tested applications use a diverse set of instructions, thus in MaRM the effective ROB size is made equal to the ROB capacity of the processor (224 entries). The exceptions are SpDMM and 2D Convolution, which are dominated by FP instructions, hence it is considered an effective ROB size around 132/140 (obtained from the micro-architecture benchmarking).

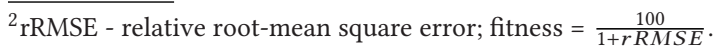



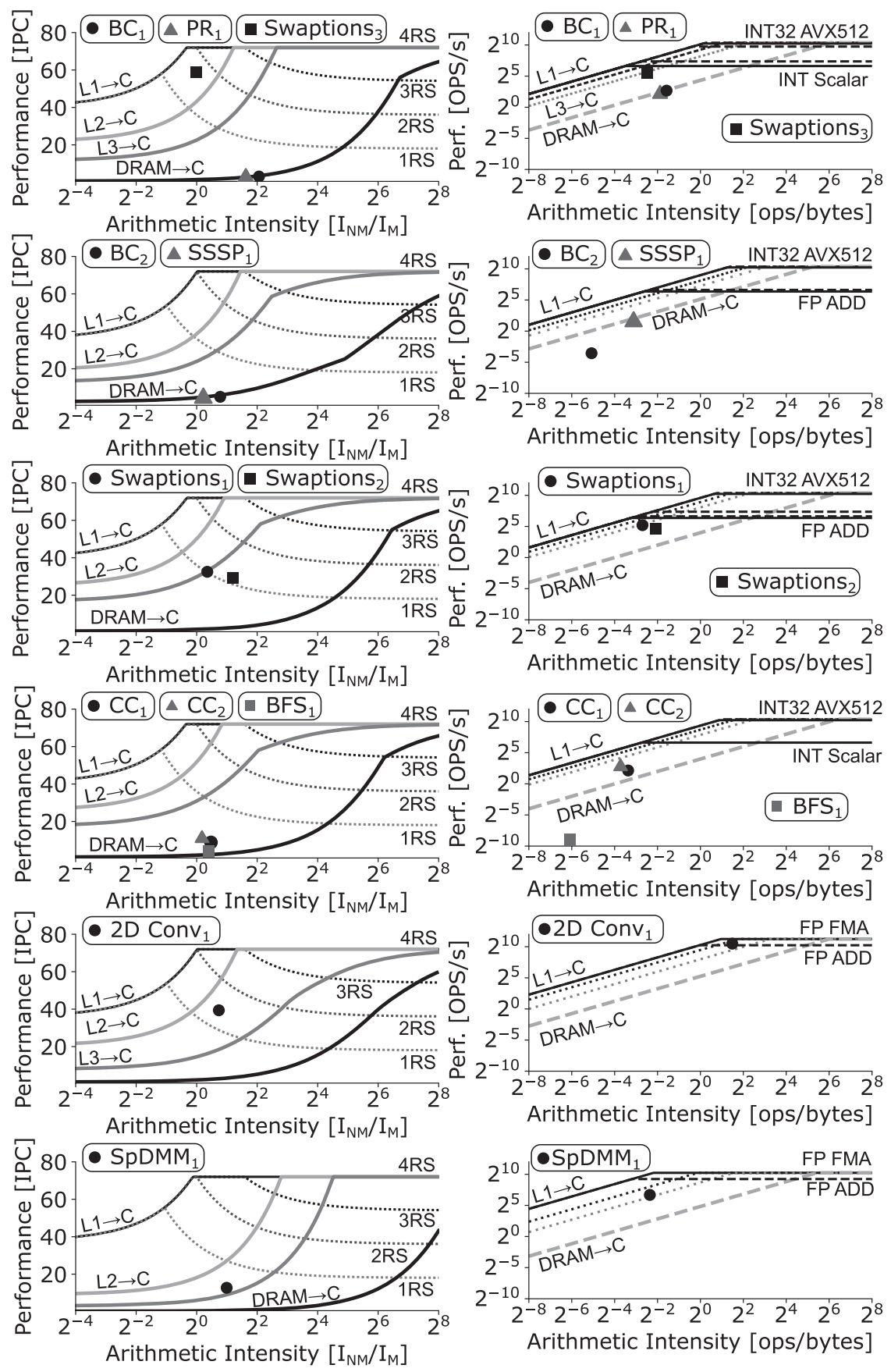

Fig. 11. Application characterization in MaRM (left) and SoA CARM (right).

To verify the characterization accuracy of CARM and MaRM, the insights provided by both models are compared with the Top-Down method by using the Intel Vtune. Top-Down classifies application bottlenecks into five main categories, namely frontend (FE), bad speculation (BS), retiring (RET), core bound (CB), and memory-bound (MB). RET and CB represent applications 
limited by the retirement rate of the micro-architecture and port utilization. FE and BS correspond to performance penalties from instruction fetch/decoding, and branch misprediction, respectively. MB highlights issues related to memory accesses. The results of the Top-Down evaluation are presented in Figure 10. In the 1st level of Top-Down (Figure 10(a)), most of the kernels are strongly limited by memory, with the exception of the Swaptions and the 2D Conv. Swaptions kernels are balanced between frontend, retiring, and memory, while 2D Conv. is balanced between retiring and memory. Since most of the kernels have a significant memory component, the memory bound level of Top-Down is also presented in Figure 10(b). As it can be observed, most of the memory accesses are limited by a mixture of DRAM Bandwidth (DRAM Bw.) and DRAM Latency (DRAM Lat.), except for Swaptions and MKL SpDMM, which are mainly limited by L1 and L3 caches, respectively. 2D Conv. also has some of its memory accesses limited by L1 and L3 caches.

The characterization of the kernels in MaRM and SoA CARM is presented in Figure 11. BFS $B C_{2}$ and Swaption $_{3}$ are differently characterized by the two models. In CARM, $B F S_{1}$ and $B C_{2}$ are placed far below the DRAM roof, pinpointing that the kernels are fully limited by the DRAM latency. However, MaRM places these kernels on top of DRAM roof and in the mixed region, hinting that these applications are limited by both DRAM latency and bandwidth, fully correlating with Top-Down. Swaptions 3 is characterized in CARM as L3 bound, while in MaRM it is placed right below the flat region corresponding to the maximum retirement rate of the micro-architecture. MaRM insights corroborate with the Top-Down method, since this kernel is mostly limited by L1 cache and retiring, not attaining maximum performance due to frontend issues.

The characterization of the kernels $C C_{1}, C C_{2}$, and $S S S P_{1}$ (Figure 11), is similar in both models, but diverging in the region where the kernels are located. In the CARM, these kernels are in the memory-bound region, while MaRM places them in the mixed region. This is supported by the Top-Down analysis, since in the mixed region of MaRM, kernels limited by DRAM are expected to partially suffer from latency issues due to the limited amount of in-flight memory requests. Similar insights can be derived for the $P R_{1}$ and $B C_{1}$ kernels. Finally, Swaptions $s_{1}$, Swaptions $2,2 D$ Conv $_{1}$, and $S p D M M_{1}$ are characterized in the same region and limited by the same bottlenecks in both models. Since these applications are mainly limited by components closer to the core, it indicates that the MaRM methodology does not lose accuracy neither correctness when characterizing these type of applications.

The improvements in characterization provided by MaRM result from the inclusion of all the instructions retired by the applications, together with the modeling of the ROB and the PRF effects on the memory subsystem. By considering all the instructions, both performance and AI are evaluated correctly from a micro-architectural point-of-view, rectifying the region and performance of the application. Moreover, by accurately modeling the memory characteristics, the DRAM bottlenecks in MaRM mixed region are intrinsically related to DRAM bandwidth and DRAM latency, allowing to correctly identify both issues. Since CARM only considers the maximum DRAM bandwidth across the entire range of AI, it is only possible to conclude from this model that kernels closer to DRAM are limited by bandwidth, independently of the kernels being in the memory or mixed regions.

\subsection{Bioinformatic Algorithm: Epistasis Detection Optimization}

To showcase the ability of MaRM to provide accurate insights when optimizing applications, an epistasis detection algorithm is optimized by following the hints provided by MaRM. This algorithm is widely used to uncover the Single-Nucleotide Polymorphism (SNP) combination that is most likely to cause a disease or trait in a given dataset. The baseline algorithm uses the approach considered in Reference [32], i.e., it contains a set of bitwise operations and population count (popcount) instructions, which are not commonly included in any of the INT or FP SoA 


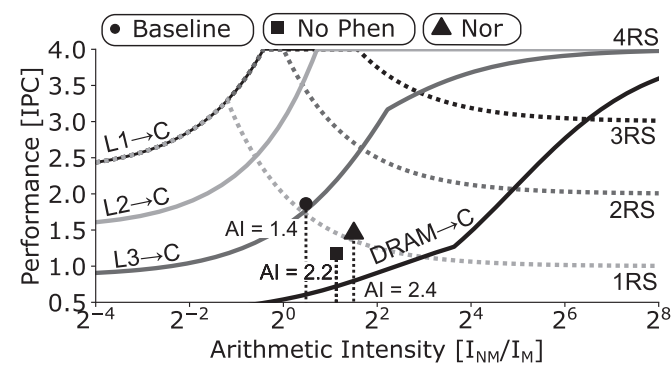

(a) MaRM characterization.

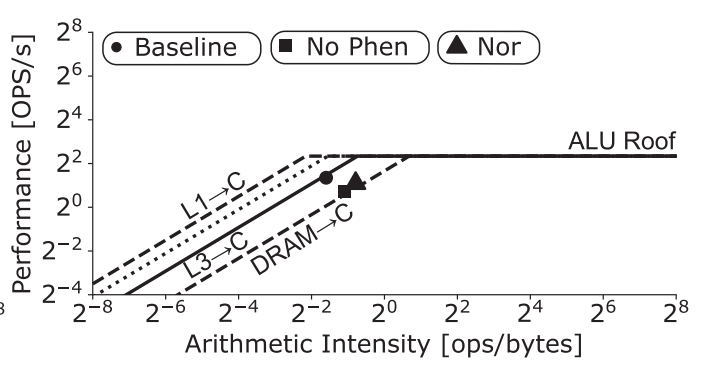

(b) CARM characterization.

Fig. 12. Characterization of the Baseline, No Phen, and Nor versions of the Epistasis Detection algorithm in MaRM and CARM.

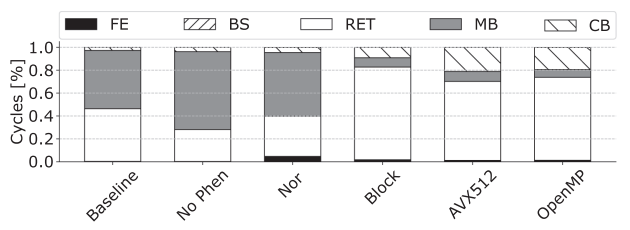

(a) 1st level breakdown.

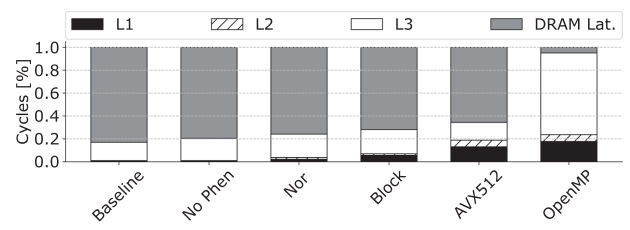

(b) Memory bound (MB) breakdown.

Fig. 13. Top-Down method of Epistasis Detection Algorithm.

roofline models. The input dataset of this algorithm is a matrix organized with the SNP in rows and patients in columns. Each Single-Nucleotide Polymorphism (SNP) is represented by three binary arrays, that express the genotypes. A phenotype is also associated with each patient, indicating if the patient has the disease (case) or does not have the disease (control). In this work, the dataset contains 10,040 SNPs and 104,448 patients, i.e., more than 50 million pairwise combinations of SNPs.

The characterization of the baseline algorithm in MaRM and CARM is presented in Figure 12(a) and (b), respectively. In both models, the baseline algorithm is mainly limited by the L3 cache and placed in the mixed region of the models, i.e., close to the compute roof of 1RS in MaRM and on top of L3 cache in CARM. These insights corroborate the results in Figure 13, obtained with the Top-Down Method, which shows that the execution is mainly limited by memory accesses and retiring. Since the application is memory bound and mainly limited by L3 cache, the user must focus on memory related optimizations, such as, improving the memory access pattern or on reducing the amount of memory accesses. This is the case of the No Phen and Nor optimizations. In the first optimization (No Phen) the phenotype is discarded by separating the dataset into cases and controls. The second optimization (Nor) only uses genotypes 0 and 1 for each patient, while genotype 2 is obtained by applying nor instructions over the two remaining genotypes. Both these optimizations allow reducing the the number of memory accesses.

As it can be observed in Figure 12(a), these optimization techniques resulted in an increase of the arithmetic intensity of the application, through the reduction of the memory instructions performed. However, it is also possible to verify that the IPC of these two versions is lower than the baseline, although the execution time reduced $1.42 \times$ for the No Phen version and $1.72 \times$ for the Nor version, in comparison to the Baseline application. This effect occurs due to the reduction of the total instructions performed by the applications. Compared to the baseline algorithm, the retired instructions for No Phen version reduced $2.27 \times$, while for Nor version this reduction was around 


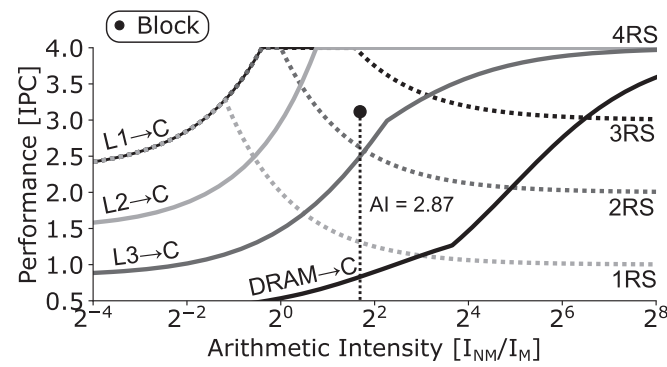

(a) MaRM characterization.

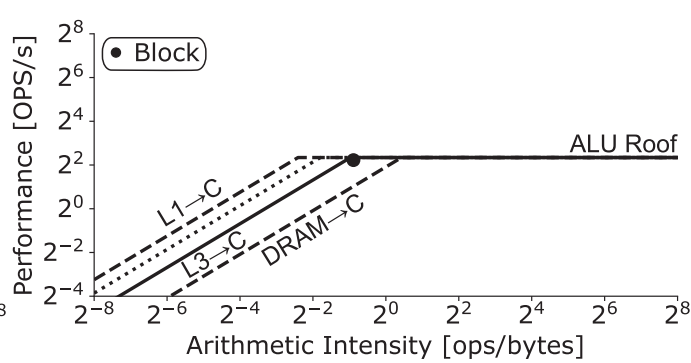

(b) CARM characterization.

Fig. 14. Characterization of the Block version of the Epistasis Detection algorithm in MaRM and CARM.

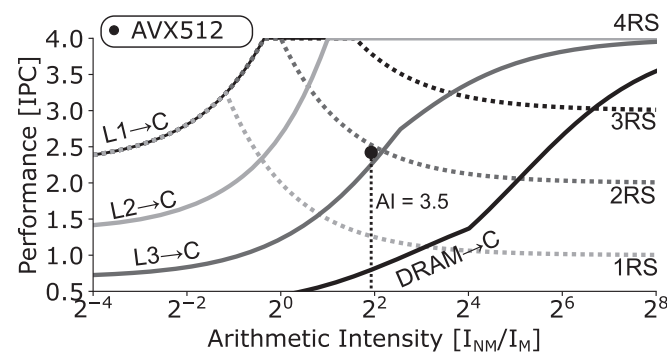

(a) MaRM characterization.

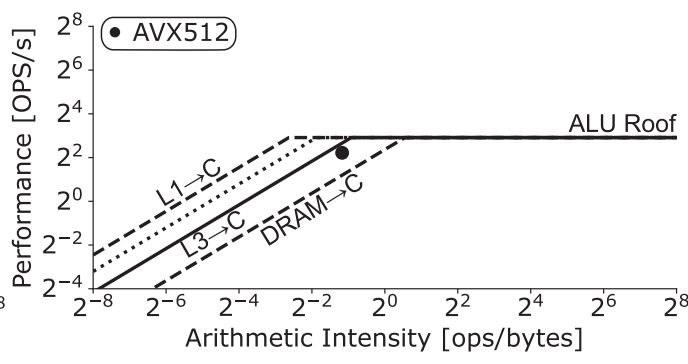

(b) CARM characterization.

Fig. 15. Characterization of the AVX512 version of the Epistasis Detection algorithm in MaRM and CARM.

$2 \times$. Since the decrease in instructions is higher than the time/cycles improvement, the applications attain a lower IPC and become more memory bound. However, this effect is not exclusive to MaRM. As it can be observed in Figure 12(b), the reduction of the number of compute operations (around $4 \times$ ) resulted in a performed drop in CARM, since the improvement of the execution time is lower than the operations reduction. For these two versions, the characterization between both models also differs. While MaRM also places the applications close to the 1RS, which is responsible for the retiring contribution according to the Top-Down method, CARM pinpoints DRAM as the main bottlenecks and does not hint any retiring contribution. Moreover, MaRM is also able to hint the DRAM latency issues pinpointed by the Top-Down analysis (Figure 13(b)) for these two application versions; the application is placed in the region around the DRAM ridge point, where the memory bandwidth is only a fraction of the maximum sustainable bandwidth of the micro-architecture.

Since the Nor application has a low retirement rate and it is placed below L3 cache roof, to boost application execution it is necessary to further improve the memory accesses. This task is performed by introducing cache blocking techniques, improving the the memory access pattern and resulting in the Block version of the application, which attained a speedup around 3.6× when compared to the Baseline version. As it can be observed in Figure 14(a), in MaRM, the Block version is placed between the compute roofs $2 \mathrm{RS}$ and 3RS, hinting its compute-bound nature. Similarly, as shown in Figure 14(b), CARM also indicates that the Block version is compute bound. TopDown provides the same hints, characterizing the application as limited by retiring, with small contributions from the core bound and the memory (in particular L3 cache and DRAM Latency as shown in Figure 13(b)).

The Block version of the application only contains scalar instructions and it is limited in the MaRM by the higher retirement roofs. Thus, it is recommended to vectorize the application, which 


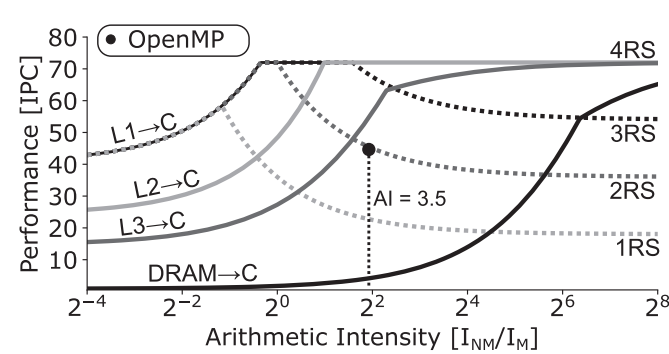

(a) MaRM characterization.

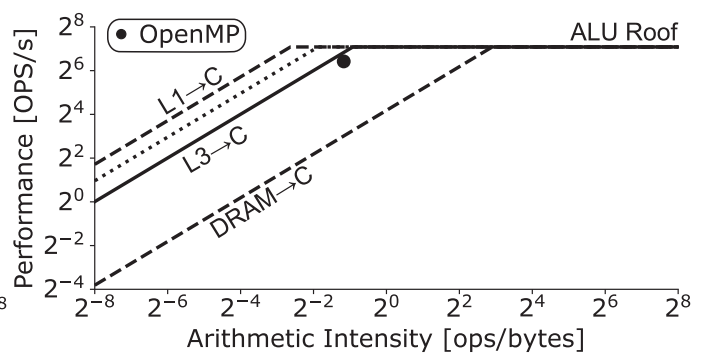

(b) CARM characterization.

Fig. 16. Characterization of the OpenMP version of the Epistasis Detection algorithm in MaRM and CARM.

is performed through the utilization of AVX512 intrinsics, allowing to attain a time speedup of 5.1× compared to the Baseline version. MaRM (Figure 15(a)) places the AVX512 version of the algorithm on top of the $2 \mathrm{RS}$ roof, indicating that it is completely limited by the retirement units, which is corroborated by the Top-Down analysis, that identifies as the main bottlenecks the core and the retirement. However, CARM characterization (Figure 15(b)) is inaccurate, since the kernel is placed below the L3 cache roof. It is important to notice that MaRM is able to accurately characterize this kernel due to its modeling approach that considers all the instructions retired by the application. Given that the Intel Xeon Gold 6140 does not support vectorized popcounts, the use of extract instructions was required, which are not accounted for in current roofline models. Additionally, it is also possible to observe a drop on the IPC between Block and AVX512 versions. This effect results from the limited number of ports that support AVX512 instruction in Skylake-SP micro-architecture. Hence, it is possible to conclude that the AVX512 algorithm is able to attain the maximum retirement of the micro-architecture for AVX512 instructions. Moreover, since the AVX512 version attained the maximum IPC allowed by AVX512 instructions, MaRM hints that the vectorization attained close to maximum efficiency.

Finally, since the single-threaded algorithm is already vectorized and completely limited by the retiring roofs in MaRM, the application is parallelized by using the OpenMP programming model. This parallel version is limited by the 2RS roof in MaRM (Figure 16(a)) and attains a speedup of $18.5 \times$ compared to the AVX512 version and $94 \times$ when compared to the baseline version. The superlinear speedup between the AVX512 and OpenMP versions results from the higher utilization of the L3 cache in the OpenMP, as it is observed in Figure 13(b). As it can be observed in Figure 16(b), CARM continues to indicate that the application is completely limited by L3 cache, which does not corroborate Top-Down analysis.

\section{RELATED WORK}

The popularity of roofline approaches results from their insightfulness and usability. For this reason, in the related SoA, several works aim at extending the capabilities of these models. The ORM is applied to Graphics Processing Units for the instruction domain in Reference [8]. However, this approach does not consider any additional issues to the modeling of micro-architecture upper bounds, as herein proposed MaRM. Cabezas et al. extends Original Roofline Model (ORM) by incorporating several roofs related with different components of the back-end of OoO CPUs [6]. The work in Reference [6] is performed by using directed acyclic graphs and micro-architectural simulations. In contrast, MaRM methodology results from accurate micro-architecture benchmarking, that allows to experimentally assess the real capabilities of the CPUs. Moreover, by representing a significant number of back-end components in a roofline chart, the model loses its simplicity 
and insightfulness. The proposed MaRM takes a simpler approach, by assessing the impact of the hardware components in the system upper bounds, maintaining simplicity while increasing its modeling accuracy.

Other SoA works focus on CARM approach. Denoyelle et al. extended CARM to support non-uniform memory accesses, by including in the memory region of the model bottlenecks related to the remote DRAM. The work in Reference [29] applies SoA CARM approach to Nvidia Graphics Processing Units. While these works maintain the modeling simplicity of CARM, none of them considers the retirement of instructions or memory latency issues. The execution-cachememory performance model (ECM) proposed in Reference [44] is a method analogous to roofline modeling approaches. ECM aims at identifying main execution bottleneck that limits application performance, by predicting the amount of cycles consumed by each memory level, assuming that cache lines are fetched at maximum throughput. However, differently from MaRM, ECM always consider maximum memory bandwidth, and does not account for the retirement issues that result from $\mathrm{ROB}$ and $\mathrm{PRF}$ when accessing high latency memory levels.

The bottlenecks related to the retirement of instructions are also tackled in several SoA works. Methods based on CPI-stacks $[13,14]$ are usually used to provide a low-level decoupling of microarchitecture bottlenecks. However, these models usually do not contain the explicit impact related to ROB size, PRF and in-flight memory requests, nor provide visual guidance of the application optimization. Both these aspects are properties of the MaRM. Jongerius et al. proposed a modeling framework to reduce the design-optimization space when targeting exascale systems [21]. This framework is mainly based on a LLVM-based application characterization and analytical modeling of the entire processor, including the retiring components. The work in Reference [45] improved the interval model [12] when predicting the performance and power consumption of applications, and also provides analytical modeling of the core pipeline together with a micro-architecture independent profile of applications. The X-model presented in Reference [26] focuses on modeling the effects of parallelism in multi-thread systems. Similarly to MaRM, it also provides a visual interpretation from performance issues that arise from memory, instruction, thread and data parallelisms. While X-model is only applied to parallel systems, MaRM can be used for both sequential or multi-thread machines and it is able to clearly pinpoint which memory levels are responsible for degrading performance. Finally, the PROFHET method proposed in Reference [35] also considers the ROB size and uses latency-bandwidth curves to model performance and power consumption of applications when relying on different memory technologies. While these works aim at predicting the behavior of applications in different hardware systems, MaRM provides a visual representation of the main bottlenecks of applications, balancing accuracy and insighfulness.

\section{CONCLUSIONS AND FUTURE WORK}

With the introduction of wider core pipelines and deeper memory levels with bigger caches and new technologies, modern processors became highly complex. For this reason, it is a challenging task to uncover the performance limits of current systems, as well as to identify the hardware components that limit application execution. To overcome these issues, roofline models can be used to quickly focus the optimization effort, due to their high-level analysis of the micro-architectures. However, these models neglect several potential bottlenecks that arise from the complexity of modern processors, providing unreachable performance upper bounds. This limits the model accuracy when characterizing real-world applications.

To tackle these issues, the MaRM proposed in this work considers all the execution units in the core pipeline and the bottlenecks related to crucial retirement components, in particular, the amount of retirement slots, Reorder-Buffer and Physical Register File sizes. The proposed model was validated with an average rRMSE of $5.4 \%$ on a real platform containing an Intel Xeon 6140 
Gold processor, while also accurately characterizing 12 kernels from real-world applications. The insights provided by the proposed model allowed to optimize a bioinformatic algorithm for epistasis detection obtaining speed-up of $5 \times$ and $18.5 \times$ when using OpenMP for multi-threading execution. Future work will focus on applying MaRM to different devices and modeling domains, i.e., power consumption and energy efficiency.

\section{REFERENCES}

[1] D. Abts, J. Ross, J. Sparling, M. Wong-VanHaren, M. Baker, T. Hawkins, A. Bell, J. Thompson, T. Kahsai, G. Kimmell, J. Hwang, R. Leslie-Hurd, M. Bye, E. R. Creswick, M. Boyd, M. Venigalla, E. Laforge, J. Purdy, P. Kamath, D. Maheshwari, M. Beidler, G. Rosseel, O. Ahmad, G. Gagarin, R. Czekalski, A. Rane, S. Parmar, J. Werner, J. Sproch, A. Macias, and B. Kurtz 2020. Think fast: A tensor streaming processor (TSP) for accelerating deep learning workloads. In Proceedings of the ACM/IEEE 47th Annual International Symposium on Computer Architecture (ISCA'20). 145-158. https://doi.org/ 10.1109/ISCA45697.2020.00023

[2] C. S. Anderson, J. Zhang, and M. Cornea. 2018. Enhanced vector math support on the Intel ${ }^{\circledR}$ AVX-512 architecture. In Proceedings of the IEEE 25th Symposium on Computer Arithmetic (ARITH'18). 120-124. https://doi.org/10.1109/ARITH. 2018.8464794

[3] Y. Bao, C. Bienia, and K. Li. 2016. The PARSEC Benchmark Suite Tutorial. Princeton University. http://parsec.cs. princeton.edu/download/tutorial/3.0/parsec-tutorial.pdf.

[4] Scott Beamer, Krste Asanović, and David Patterson. 2015. The GAP benchmark suite. CoRR abs/1508.03619. arXiv:1508.03619. http://arxiv.org/abs/1508.03619.

[5] Natalie N. Beams, Adrianna Gillman, and Russell J. Hewett. 2020. A parallel shared-memory implementation of a high-order accurate solution technique for variable coefficient Helmholtz problems. Comput. Math. Appl. 79, 4 (2020), 996-1011.

[6] Victoria Caparrós Cabezas and Markus Püschel. 2014. Extending the roofline model: Bottleneck analysis with microarchitectural constraints. In Proceedings of the International Symposium on Workload Characterization. IEEE, 222-231.

[7] Salvatore Cielo, Luigi Iapichino, Fabio Baruffa, Matteo Bugli, and Christoph Federrath. 2020. Honing and proofing astrophysical codes on the road to exascale. experiences from code modernization on many-core systems. Fut. Gener. Comput. Syst. 112 (2020), 93-107. https://doi.org/10.1016/j.future.2020.05.003

[8] Nan Ding and Samuel Williams. 2019. An instruction roofline model for gpus. In Proceedings of the International Workshop on Performance Modeling, Benchmarking and Simulation of High Performance Computer Systems. IEEE, $7-18$.

[9] Douglas Doerfler, Jack Deslippe, Samuel Williams, Leonid Oliker, Brandon Cook, Thorsten Kurth, Mathieu Lobet, Tareq Malas, Jean-Luc Vay, and Henri Vincenti. 2016. Applying the roofline performance model to the intel xeon phi knights landing processor. In Proceedings of the International Conference on High Performance Computing. Springer, 339-353.

[10] Daniel Drzisga, Ulrich Rüde, and Barbara Wohlmuth. 2019. Stencil scaling for vector-valued PDEs with applications to generalized newtonian fluids. CoRR abs/1908.08666. arXiv:1908.08666 http://arxiv.org/abs/1908.08666.

[11] V. Etienne, T. Tonellot, K. Akbudak, H. Ltaief, S. Kortas, T. Malas, P. Thierry, and D. Keyes. 2018. Optimization of finitedifference kernels on multi-core architectures for seismic applications. In Intel eXtreme Performance Users Group.

[12] Stijn Eyerman, Lieven Eeckhout, Tejas Karkhanis, and James E. Smith. 2009. A mechanistic performance model for superscalar out-of-order processors. ACM Trans. Comput. Syst. 27, 2 (2009), 1-37.

[13] S. Eyerman, W. Heirman, K. Du Bois, and I. Hur. 2018. Extending the performance analysis tool box: Multi-stage CPI stacks and FLOPS stacks. In Proceedings of the IEEE International Symposium on Performance Analysis of Systems and Software (ISPASS'18). 179-188. https://doi.org/10.1109/ISPASS.2018.00031

[14] S. Eyerman, K. Hoste, and L. Eeckhout. 2011. Mechanistic-empirical processor performance modeling for constructing CPI stacks on real hardware. In Proceedings of the IEEE International Symposium on Performance Analysis of Systems and Software (ISPASS'11). 216-226. https://doi.org/10.1109/ISPASS.2011.5762738

[15] Andrei Frumusanu. 2020. Apple Announces The Apple Silicon M1: Ditching x86-What to Expect, Based on A14. Retrieved from https:/www.anandtech.com/show/16226/apple-silicon-m1-a14-deep-dive.

[16] Philipp Grete, Forrest W. Glines, and Brian W. O'Shea. 2020. K-athena: A performance portable structured grid finite volume magnetohydrodynamics code. IEEE Trans. Parallel Distrib. Syst. 32, 1 (2021), 85-97. https://doi.org/10.1109/ TPDS.2020.3010016

[17] Mark Hill and Vijay Janapa Reddi. 2019. Gables: A roofline model for mobile socs. In Proceedings of the IEEE International Symposium on High Performance Computer Architecture (HPCA'19). IEEE, 317-330.

[18] Aleksandar Ilic, Frederico Pratas, and Leonel Sousa. 2013. Cache-aware roofline model: Upgrading the loft. IEEE Comput. Arch. Lett. 13, 1 (2013), 21-24. 
[19] Bruce Jacob. 2009. The memory system: You can't avoid it, you can't ignore it, you can't fake it. Synth. Lect. Comput. Arch. 4, 1 (2009), 1-77.

[20] Yangqing Jia, Evan Shelhamer, Jeff Donahue, Sergey Karayev, Jonathan Long, Ross Girshick, Sergio Guadarrama, and Trevor Darrell. 2014. Caffe: Convolutional architecture for fast feature embedding. In Proceedings of the 22nd ACM International Conference on Multimedia (Orlando, Florida, USA) (MM'14). Association for Computing Machinery, New York, NY, USA, 675-678. https://doi.org/10.1145/2647868.2654889

[21] Rik Jongerius, Andreea Anghel, Gero Dittmann, Giovanni Mariani, Erik Vermij, and Henk Corporaal. 2017. Analytic multi-core processor model for fast design-space exploration. IEEE Trans. Comput. 67, 6 (2017), 755-770.

[22] Norman P. Jouppi, Cliff Young, Nishant Patil, David Patterson, Gaurav Agrawal, Raminder Bajwa, Sarah Bates, Suresh Bhatia, Nan Boden, Al Borchers, Rick Boyle, Pierre-luc Cantin, Clifford Chao, Chris Clark, Jeremy Coriell, Mike Daley, Matt Dau, Jeffrey Dean, Ben Gelb, Tara Vazir Ghaemmaghami, Rajendra Gottipati, William Gulland, Robert Hagmann, C. Richard Ho, Doug Hogberg, John Hu, Robert Hundt, Dan Hurt, Julian Ibarz, Aaron Jaffey, Alek Jaworski, Alexander Kaplan, Harshit Khaitan, Daniel Killebrew, Andy Koch, Naveen Kumar, Steve Lacy, James Laudon, James Law, Diemthu Le, Chris Leary, Zhuyuan Liu, Kyle Lucke, Alan Lundin, Gordon MacKean, Adriana Maggiore, Maire Mahony, Kieran Miller, Rahul Nagarajan, Ravi Narayanaswami, Ray Ni, Kathy Nix, Thomas Norrie, Mark Omernick, Narayana Penukonda, Andy Phelps, Jonathan Ross, Matt Ross, Amir Salek, Emad Samadiani, Chris Severn, Gregory Sizikov, Matthew Snelham, Jed Souter, Dan Steinberg, Andy Swing, Mercedes Tan, Gregory Thorson, Bo Tian, Horia Toma, Erick Tuttle, Vijay Vasudevan, Richard Walter, Walter Wang, Eric Wilcox, Eric Wilcox, and Doe Hyun Yoon 2017. In-datacenter performance analysis of a tensor processing unit. In Proceedings of the 44th Annual International Symposium on Computer Architecture. 1-12.

[23] L. Ke, U. Gupta, B. Y. Cho, D. Brooks, V. Chandra, U. Diril, A. Firoozshahian, K. Hazelwood, B. Jia, H. S. Lee, M. Li, B. Maher, D. Mudigere, M. Naumov, M. Schatz, M. Smelyanskiy, X. Wang, B. Reagen, C. Wu, M. Hempstead, and X. Zhang. 2020. RecNMP: Accelerating personalized recommendation with near-memory processing. In Proceedings of the ACM/IEEE 47th Annual International Symposium on Computer Architecture (ISCA'20). 790-803. https://doi.org/10. 1109/ISCA45697.2020.00070

[24] Tuomas Koskela, Jack Deslippe, Brian Friesen, and Karthik Raman. 2017. Fusion PIC code performance analysis on the cori KNL system. In Proceedings of the Cray User Group Conference.

[25] Tuomas Koskela, Zakhar Matveev, Charlene Yang, Adetokunbo Adedoyin, Roman Belenov, Philippe Thierry, Zhengji Zhao, Rahulkumar Gayatri, Hongzhang Shan, Leonid Oliker, Jack Deslippe, Ron Green, and Samuel Williams. 2018 A novel multi-level integrated roofline model approach for performance characterization. In Proceedings of the International Conference on High Performance Computing. Springer, 226-245.

[26] A. Li, S. L. Song, E. Brugel, A. Kumar, D. Chavarría-Miranda, and H. Corporaal. 2016. X: A comprehensive analytic model for parallel machines. In Proceedings of the IEEE International Parallel and Distributed Processing Symposium (IPDPS'16). 242-252. https://doi.org/10.1109/IPDPS.2016.89

[27] Jiajia Li, Mahesh Lakshminarasimhan, Xiaolong Wu, Ang Li, Catherine Olschanowsky, and Kevin Barker. 2020. A sparse tensor benchmark suite for CPUs and GPUs. In Proceedings of the IEEE International Symposium on Workload Characterization (IISWC'20). IEEE, 193-204.

[28] John D. C. Little. 1961. A proof for the queuing formula: L= $\lambda$ W. Operat. Res. 9, 3 (1961), 383-387.

[29] André Lopes, Frederico Pratas, Leonel Sousa, and Aleksandar Ilic. 2017. Exploring GPU performance, power and energy-efficiency bounds with cache-aware roofline modeling. In Proceedings of the IEEE International Symposium on Performance Analysis of Systems and Software (ISPASS'17). IEEE, 259-268.

[30] Diogo Marques, Aleksandar Ilic, Zakhar A. Matveev, and Leonel Sousa. 2020. Application-driven cache-aware roofline model. Fut. Gener. Comput. Syst. 107 (2020), 257-273.

[31] Amrita Mathuriya, Ye Luo, Raymond C. Clay III, Anouar Benali, Luke Shulenburger, and Jeongnim Kim. 2017. Embracing a new era of highly efficient and productive quantum monte carlo simulations. In Proceedings of the International Conference for High Performance Computing, Networking, Storage and Analysis. ACM, 1-12.

[32] Ricardo Nobre, Aleksandar Ilic, Sergio Santander-Jiménez, and Leonel Sousa. 2020. Exploring the binary precision capabilities of tensor cores for epistasis detection. In Proceedings of the IEEE International Parallel and Distributed Processing Symposium (IPDPS'20). IEEE, 338-347.

[33] Irma Esmer Papazian. 2020. New 3rd gen intel ${ }^{\circledR}$ xeon ${ }^{\circledR}$ scalable processor (codename: Ice lake-SP). In Proceedings of the IEEE Hot Chips 32 Symposium (HCS'20). IEEE Computer Society, 1-22.

[34] A. Pellegrini, N. Stephens, M. Bruce, Y. Ishii, J. Pusdesris, A. Raja, C. Abernathy, J. Koppanalil, T. Ringe, A. Tummala, J. Jalal, M. Werkheiser, and A. Kona. 2020. The arm neoverse N1 platform: Building blocks for the next-gen cloud-toedge infrastructure SoC. IEEE Micro 40, 2 (2020), 53-62.

[35] Milan Radulovic, Rommel Sánchez Verdejo, Paul Carpenter, Petar Radojković, Bruce Jacob, and Eduard Ayguadé. 2019. PROFET: Modeling system performance and energy without simulating the CPU. SIGMETRICS Perform. Eval. Rev. 47, 1 (Dec. 2019), 71-72. https://doi.org/10.1145/3376930.3376976 
[36] N. Srivastava, H. Jin, J. Liu, D. Albonesi, and Z. Zhang. 2020. MatRaptor: A sparse-sparse matrix multiplication accelerator based on row-wise product. In Proceedings of the 53rd Annual IEEE/ACM International Symposium on Microarchitecture (MICRO'20). 766-780. https://doi.org/10.1109/MICRO50266.2020.00068

[37] N. Srivastava, H. Jin, S. Smith, H. Rong, D. Albonesi, and Z. Zhang. 2020. Tensaurus: A versatile accelerator for mixed sparse-dense tensor computations. In Proceedings of the IEEE International Symposium on High Performance Computer Architecture (HPCA'20). 689-702. https://doi.org/10.1109/HPCA47549.2020.00062

[38] JEDEC Standard. 2012. DDR4 SDRAM. FEDEC Solid State Technology Association, JESD79-4 151 (2012).

[39] JEDEC Standard. 2013. High bandwidth memory (HBM) DRAM. JESD235 (2013).

[40] JEDEC Standard. 2020. DDR5 SDRAM. FEDEC Solid State Technology Association, JESD79-5 (2020).

[41] David Suggs, Mahesh Subramony, and Dan Bouvier. 2020. The AMD “Zen 2" processor. IEEE Micro 40, 2 (2020), 45-52. https://doi.org/10.1109/MM.2020.2974217

[42] Ady Tal. 2012. Intel® Software Development Emulator. Retrieved from https://software.intel.com/en-us/articles/intelsoftware-development-emulator.

[43] Sofya Titarenko and Mark Hildyard. 2017. Hybrid multicore/vectorisation technique applied to the elastic wave equation on a staggered grid. Comput. Phys. Commun. 216 (2017), 53-62.

[44] Jan Treibig and Georg Hager. 2009. Introducing a performance model for bandwidth-limited loop kernels. In Proceedings of the International Conference on Parallel Processing and Applied Mathematics. Springer, 615-624.

[45] Sam Van den Steen, Sander De Pestel, Moncef Mechri, Stijn Eyerman, Trevor Carlson, David Black-Schaffer, Erik Hagersten, and Lieven Eeckhout. 2015. Micro-architecture independent analytical processor performance and power modeling. In Proceedings of theInternational Symposium on Performance Analysis of Systems and Software (ISPASS'15). IEEE, 32-41.

[46] Xavier Vera. 2020. Inside tiger lake: Intel's next generation mobile client CPU. In Proceedings of the IEEE Hot Chips 32 Symposium (HCS'20). IEEE Computer Society, 1-26.

[47] Endong Wang, Qing Zhang, Bo Shen, Guangyong Zhang, Xiaowei Lu, Qing Wu, and Yajuan Wang. 2014. Intel math kernel library. In HighPerformance Computing on the Intel $\circledast^{X e o n}$ Phi $^{T M}$. Springer, 167-188. https://doi.org/10.1007/9783-319-06486-4_7

[48] Samuel Williams, Andrew Waterman, and David Patterson. 2009. Roofline: An insightful visual performance model for multicore architectures. Commun. ACM 52, 4 (2009), 65-76. https://doi.org/10.1145/1498765.1498785

[49] Henry Wong. 2013. Measuring Reorder Buffer Capacity. Retrieved from http://blog.stuffedcow.net/2013/05/measuringrob-capacity/.

[50] Ahmad Yasin. 2014. A top-down method for performance analysis and counters architecture. In Proceedings of the International Symposium on Performance Analysis of Systems and Software. IEEE, 35-44.

Received April 2021; revised July 2021; accepted July 2021 